\title{
Association Between Constipation and Frailty Components in Patients Undergoing Late Phase II Cardiac Rehabilitation
}

\author{
Miho Nishitani-Yokoyamaa, b, e, Kazunori Shimada ${ }^{a}$, ${ }^{\text {, Miki Yamada }}{ }^{\mathrm{b}}$, Akio Honzawa $^{\mathrm{b}}$, \\ Mitsuhiro Kunimoto $^{a}$, Yurina Sugita ${ }^{a}$, Kei Fujiwara ${ }^{a}$, Tomomi Matsubara ${ }^{a}$, Rie Matsumoria ${ }^{a}$, \\ Abidan Abulimiti ${ }^{\mathrm{a}}$, Akie Shimada ${ }^{\mathrm{c}}$, Taira Yamamotoc, Tohru Asaic, Atsushi Amanoc, \\ Masakazu Saitoh ${ }^{\mathrm{d}}$, Tomoyuki Morisawa ${ }^{\mathrm{d}}$, Tetsuya Takahashid, \\ Hiroyuki Daida ${ }^{\text {a, }}$, Tohru Minamino ${ }^{\text {a }}$
}

\begin{abstract}
Background: The Japanese Ministry of Health, Labour and Welfare reported that the overall constipation complaint rates among men and women are $2.5 \%$ and $4.6 \%$, respectively. To evaluate the impact of constipation on patients with cardiovascular diseases, we investigated the association between constipation and frailty components in patients undergoing cardiac rehabilitation (CR).

Methods: We enrolled 102 consecutive patients undergoing late phase II CR (mean age: $62.7 \pm 13.4$ years; 68 (67\%) were men). We investigated clinical characteristics, observed defecation status and evaluated frailty components assessed by the Kihon checklist. According to the Clinical Guidelines for Chronic Constipation, the subjects were divided into constipation and non-constipation groups.

Results: Constipation was noted in 33 patients (32\%). Interestingly, the constipation complaint rate was only $15 \%$. Age was significantly higher in the constipation group than in the non-constipation group; however, no differences in sex, underlying diseases and prevalence rates of coronary risk factors were observed between the two groups. Body mass index, hemoglobin level, albumin level and estimated glomerular filtration rate were significantly lower in the constipation group than in the non-constipation group. The prevalence of frailty
\end{abstract}

Manuscript submitted April 3, 2021, accepted April 17, 2021

Published online May 14, 2021

aDepartment of Cardiovascular Biology and Medicine, Juntendo University Graduate School of Medicine, Tokyo, Japan

${ }^{\mathrm{b}}$ Cardiovascular Rehabilitation and Fitness, Juntendo University Hospital, Tokyo, Japan

'Department of Cardiovascular Surgery, Juntendo University Graduate School of Medicine, Tokyo, Japan

dJuntendo University, Faculty of Health Science, Tokyo, Japan

${ }^{\text {e}}$ Corresponding Author: Miho Nishitani-Yokoyama, Department of Cardiovascular Biology and Medicine, Juntendo University Graduate School of Medicine, 2-1-1, Hongo, Bunkyo-ku, Tokyo 113-8421, Japan.

Email: mipocch@juntendo.ac.jp

doi: https://doi.org/10.14740/cr1246 was significantly higher in the constipation group than in the nonconstipation group. Physical ability, seclusion and depression scores were significantly higher in the constipation group than the non-constipation group.

Conclusions: Constipation was associated with physical function and depression score as components of frailty in patients undergoing CR.

Keywords: Constipation; Defecation; Cardiovascular disease; Frailty; Cardiac rehabilitation

\section{Introduction}

Constipation is one of the most common gastrointestinal disorders, with a reported prevalence of about $20 \%$, ranging from $2 \%$ to $28 \%$ globally, depending on diagnostic criteria [1]. According to the 2016 comprehensive survey of living conditions conducted by the Japanese Ministry of Health, Labour and Welfare, the prevalence of constipation in Japan was 2-5\%, with more women being affected than men $(4.6 \%$ vs. $2.5 \%)$ [2]. Breath-holding methods like Valsalva maneuver as a result of straining associated with defecation can cause changes in circulation and increase rupture risks of intracranial aneurysms [3]. However, the prevalence of constipation in patients with cardiovascular disease (CVD) is not known.

In 2017, the Clinical Guidelines for Chronic Constipation was prepared by the Constipation Diagnosis and Treatment Study Group of the Japan Society of Gastroenterology [4], and constipation was defined as a decrease in the frequency of bowel movements, having hard stools and a defecation disorder. According to the diagnostic criteria, constipation cannot be diagnosed simply by the single factor of infrequent bowel movements but requires other symptoms such as straining during bowel movement and the consistency of the stools. Even if an individual has a sufficient number of bowel movements, there are multiple symptoms of defecation disorders that would be diagnosed as constipation.

This study investigated the complaints and prevalence of 
constipation according to the Clinical Guidelines for Chronic Constipation in patients undergoing cardiac rehabilitation (CR). Further, we also investigated the association between constipation and frailty components.

\section{Materials and Methods}

\section{Study protocol and subjects}

In 2012, the Japanese Circulation Society described the candidates for CR as being patients with CVD who have had a coronary artery disease such as acute myocardial infarction and angina pectoris, open-heart surgery, chronic heart failure, major vessel disease such as that occurring after aortic dissection, macrovascular surgery, stent graft insertion, peripheral artery disease and transcatheter aortic valve implantation [5]. $\mathrm{CR}$ is a comprehensive secondary prevention program that includes not only exercise therapy but also education for patients and their families, guidance for proper nutrition, medication, lifestyle, and smoking cessation, support for psychosocial factors, and reinstatement training [5]. The effectiveness of CR for patients with CVD has been proven [6].

Two hundred and ninety-eight consecutive patients participated in late phase II CR at Juntendo University Hospital, Tokyo, Japan, between June 2018 and June 2019. The present study enrolled 121 consecutive patients with acute myocardial infarction, chronic heart failure and major vessel disease undergoing late phase II CR. After excluding five patients who did not provide written informed consent and 14 patients with missing clinical data, we enrolled 102 patients for this analysis. The purpose of the study was explained in writing to the patients and consent was obtained in accordance with the Declaration of Helsinki. Approval was obtained for research involving patients from the Institutional Review Board of Juntendo University Hospital (approval number 17-307).

\section{Diagnostic criteria for functional constipation}

Our constipation questionnaire was prepared according to the Clinical Guidelines for Chronic Constipation [4]. We surveyed patients using six questions on defecation status based on the Clinical Guidelines for Chronic Constipation, and four questions on defecation status (constipation, diarrhea, normal, constipation and diarrhea) using a questionnaire [7]. According to the Clinical Guidelines for Chronic Constipation, constipation was diagnosed if two or more of the following symptoms have been present for at least 3 months (with symptom onset at least 6 months prior to diagnosis) [4].

The severity of constipation was determined using the Bristol Stool Form Scale (BSFS) [8], which classifies stools into seven morphologic types based on appearance. These seven morphologic types are divided into three categories. Stools associated with constipation are type 1 (separate hard lumps, like nuts and hard to pass) and type 2 (sausage-shaped, but lumpy); the ideal stools are type 3 (like sausage, but with cracks on surface), type 4 (like sausage or snake, smooth and soft), and type 5 (soft blobs with clear cut edges, passed early); stools associated with diarrhea are type 6 (fluffy pieces with ragged edges, mushy stool) and type 7 (watery, no solid pieces).

\section{The Kihon checklist (KCL) for frailty}

The KCL is a 25 -item self-administered questionnaire developed by the Japanese Ministry of Health, Labour and Welfare for older individuals to evaluate their own life and health condition and check whether their physical and mental functions are impaired. It helps in preventing the deterioration of the condition by identifying at an early stage those older individuals who may experience decline in living function and connecting it to the prevention of long-term care [9]. It consists of 25 simple "yes" or "no" questions of seven types that focus on social and psychological aspects in addition to the physical aspects of frailty and evaluates instrumental activities of daily living, physical function, nutritional status, oral function, social activities of daily living, cognitive function and depression. The KCL is considered to be an effective screening tool as it can evaluate frailty on the basis of multiple domains [9, 10]. The response to each "yes" or "no" question will receive a respective score of 1 point or 0 point, with a score range of 4 - 7 suggesting pre-frailty and a score of more than 8 points suggesting frailty [9].

\section{Data collection}

The patients' clinical characteristics (age, gender, comorbidities and medical history) were obtained from medical records. Laboratory test results, dosage of medications and echocardiographic data were used from the time closest to the initiation of CR when multiple data were available for one patient. Blood samples were taken in the early morning after fasting overnight. Chronic kidney disease was defined as an estimated glomerular filtration rate $<60 \mathrm{~mL} / \mathrm{min} / 1.73 \mathrm{~m}^{2}$ and was calculated by the renal disease equation using baseline serum creatinine level as assessed by sex and age [11].

We used the Geriatric Nutritional Risk Index (GNRI) data [12] and assessed the body composition at the beginning of the $\mathrm{CR}$, as described previously [6]. Anthropometric parameters, including the percentages of body fat, and lean body weight were measured by bioelectrical impedance analysis (MC780A; TANITA, Tokyo, Japan).

\section{Statistical analysis}

The results were expressed as mean \pm standard deviation and were analyzed using JMP software (Version 14 for Windows, SAS Institute, Inc., Cary, NC, USA). We performed comparative analyses between the constipation and non-constipation groups using a two-tailed Student's $t$-test. A P-value of less than 0.05 was considered significant. 


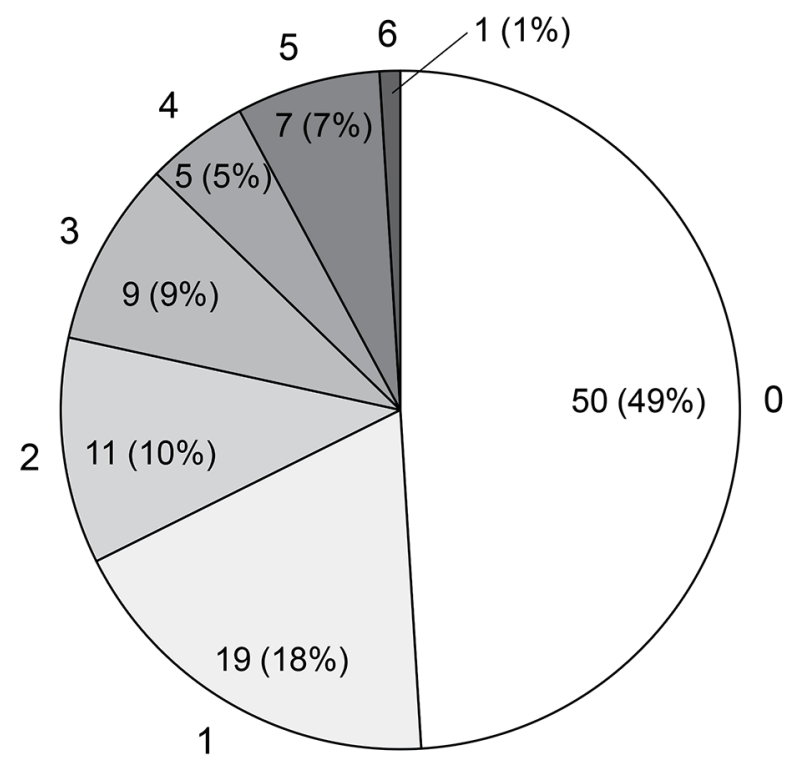

Figure 1. The number of applicable items for functional constipation diagnosis criteria. Subjects were classified into constipation $(n=33$, $32 \%$ ) and non-constipation ( $n=69,68 \%$ ) groups according to the Chronic Constipation Treatment Guidelines.

\section{Results}

\section{Study population and the complaint and prevalence rates of constipation}

The average age of the patients was $62.7 \pm 13.4$ years, and 68 $(67 \%)$ were men. Sixty-four $(63 \%)$ had chronic heart failure, $22(21 \%)$ had aortic disease, $16(16 \%)$ had acute myocardial infarction, $59(58 \%)$ had hypertension, $12(12 \%)$ had diabetes mellitus and 30 (29\%) had dyslipidemia.
Of the 102 subjects, the so-called "complaint rate" included only 15 patients (15\%) who answered to the question "I think I have constipation", but the prevalence rate of constipation included 33 patients $(32 \%)$, based on responses to our questionnaire (Figs. 1, 2). The constipation prevalence rate was significantly higher than that of the complaint rate $(\mathrm{P}<$ $0.0001)$. Twenty-one (64\%) of the 33 diagnosed with constipation were diagnosed with chronic constipation (Fig. 2).

\section{Comparison of clinical characteristics between the consti- pation and non-constipation groups}

Subjects were classified into constipation ( $\mathrm{n}=33,32 \%)$ and non-constipation $(\mathrm{n}=69,68 \%)$ groups according to the Chronic Constipation Treatment Guidelines [4], and the subjects' baseline clinical characteristics and medication use are presented in Table 1. The constipation group was older than the non-constipation group $(71.5 \pm 11.2$ vs. $58.5 \pm 12.4$ years; $\mathrm{P}<0.01)$. There was no significant difference between the two groups in sex, prevalence rate of coronary risk factors, CVD at the beginning of $\mathrm{CR}$, ejection fraction levels, or medications (except for laxatives).

Body mass index $\left(22.8 \pm 3.3\right.$ vs. $24.5 \pm 4.7 \mathrm{~kg} / \mathrm{m}^{2} ; \mathrm{P}<$ $0.05)$, levels of hemoglobin $(12.3 \pm 1.8$ vs. $13.4 \pm 2.0 \mathrm{~g} / \mathrm{dL} ; \mathrm{P}$ $<0.01)$ and albumin $(3.6 \pm 0.4$ vs. $3.9 \pm 0.4 \mathrm{~g} / \mathrm{dL} ; \mathrm{P}<0.01)$, estimated glomerular filtration rate $(50.7 \pm 20.2$ vs. $63.8 \pm$ $\left.19.3 \mathrm{~mL} / \mathrm{min} / 1.73 \mathrm{~m}^{2} ; \mathrm{P}<0.01\right)$ and Geriatric Nutritional Risk Index (GNRI) $(97.1 \pm 10.7$ vs. $103.3 \pm 12.8 ; \mathrm{P}<0.05)$ were significantly lower in the constipation group than in the nonconstipation group.

Thirty-one of the study subjects (30\%) took laxatives, with a higher prescription rate in the constipation group than in the non-constipation group ( $48 \%$ vs. $22 \%$; $\mathrm{P}<0.01)$. In addition, significantly more patients in the constipation group compared to the non-constipation group used irritant laxatives

\section{Complaint rates}

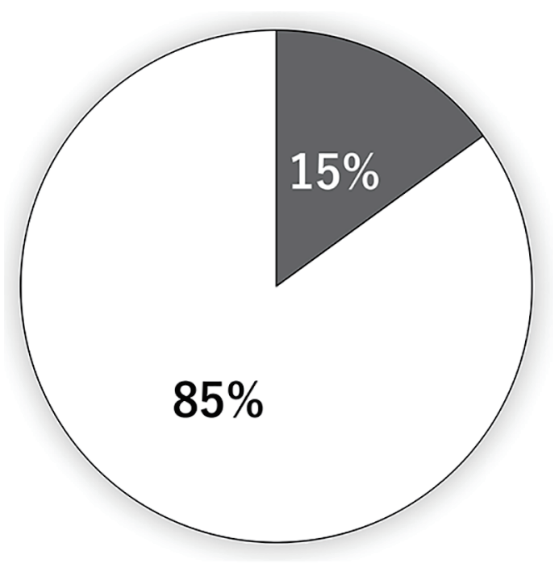

: Yes

\section{Prevalence of constipation}

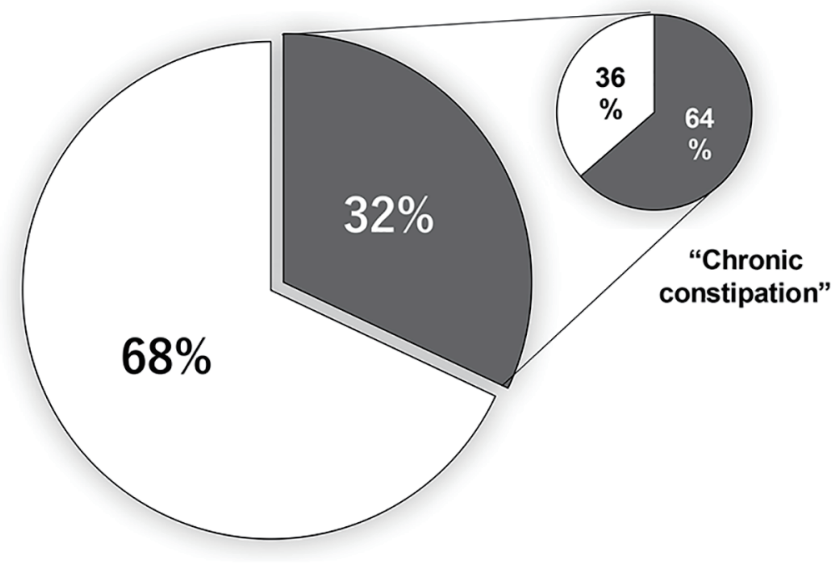

$\mathrm{P}<.0001$

Figure 2. Comparison of constipation prevalence and complaint rates in patients undergoing late phase II cardiac rehabilitation. 
Table 1. Clinical Characteristics of the Study Subjects

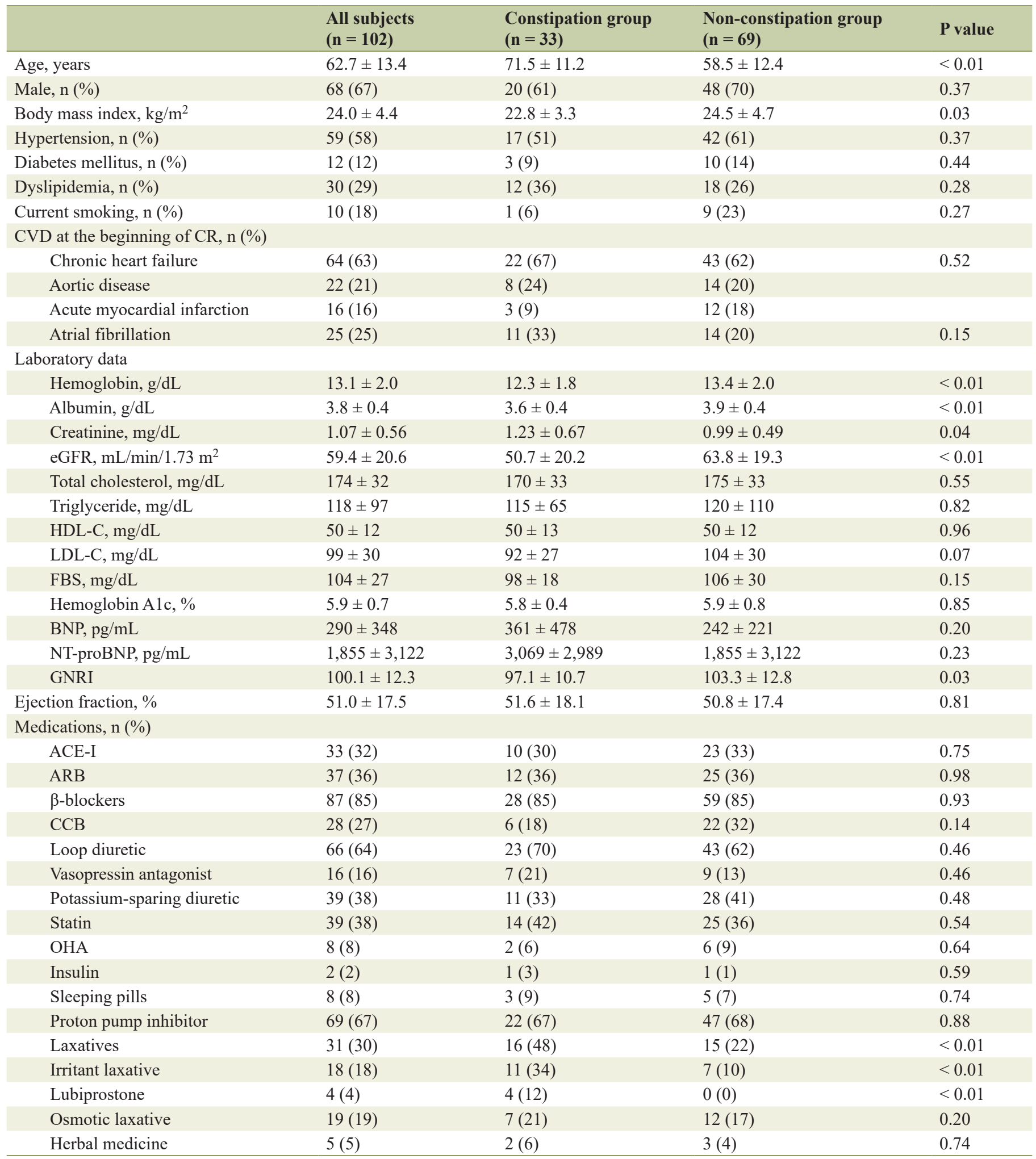

Data are presented as the mean \pm SD. CVD: cardiovascular disease; CR: cardiac rehabilitation; eGFR: estimated glomerular filtration rate; HDL-C: high-density lipoprotein cholesterol; LDL-C: low-density lipoprotein cholesterol; FBS: fasting blood sugar; BNP: brain natriuretic peptide; NT-proBNP: N-terminal pro brain natriuretic peptide; GNRI: Geriatric Nutritional Risk Index; ACE-I: angiotensin-converting enzyme inhibitor; ARB: angiotensin II receptor blocker; CCB: calcium-channel blocker; OHA: oral hypoglycemic agent. 
Table 2. Comparison of Frailty Components Between the Constipation and Non-Constipation Groups

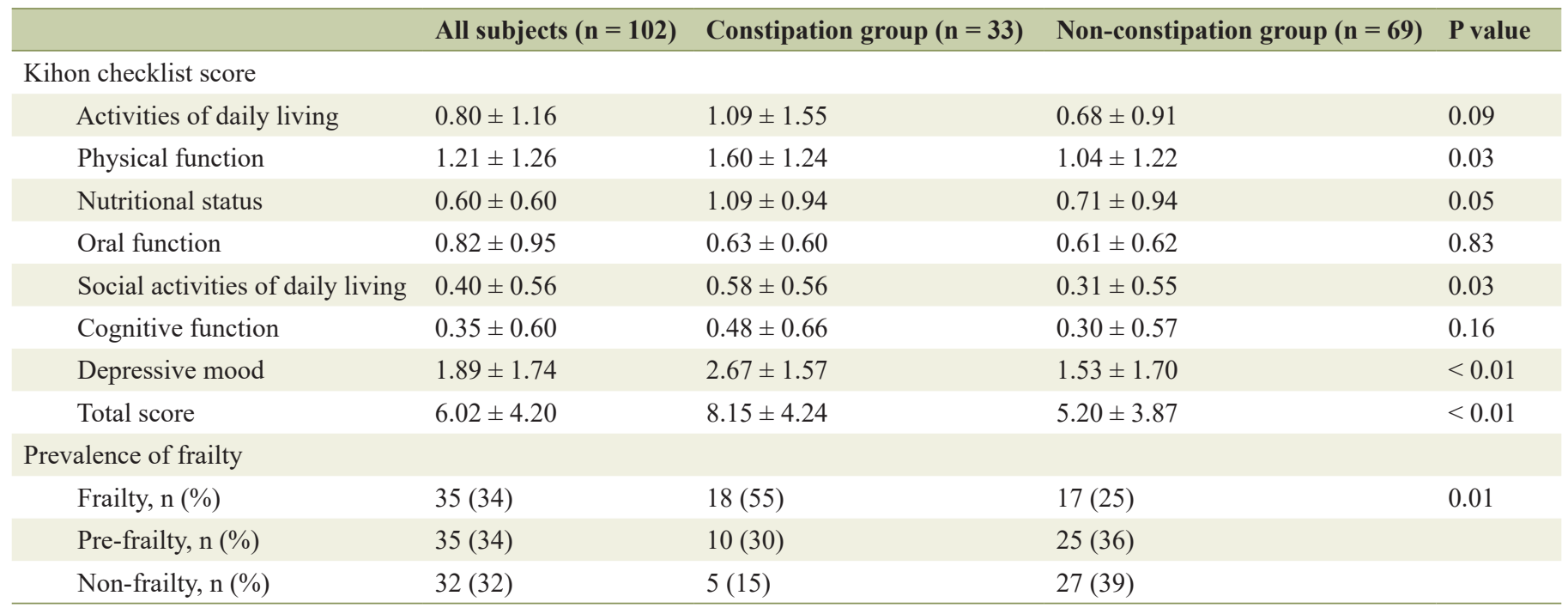

Data are presented as the mean $\pm \mathrm{SD}$.

and a new constipation drug (34\% vs. $10 \%$ and $12 \%$ vs. $0 \%$, respectively; $\mathrm{P}<0.01$ ), but no significant difference was observed between the groups in the use of osmotic laxatives $(7$ $(21 \%)$ vs. $12(17 \%) ; \mathrm{P}=0.20)$ and herbal medicine $(2(6 \%)$ vs. $3(4 \%) ; \mathrm{P}=0.74)$ (Table 1$)$.

\section{Comparison of frailty components between the constipa- tion and non-constipation groups}

Table 2 compares the frailty components of the two groups. The constipation group had significantly higher KCL scores than the non-constipation group: physical function ( $1.60 \pm 1.24$ vs. 1.04 $\pm 1.22, \mathrm{P}<0.05)$, social activities of daily life $(0.58 \pm 0.56 \mathrm{vs}$. $0.31 \pm 0.55, \mathrm{P}<0.05)$ and depression $(2.67 \pm 1.57$ vs. $1.53 \pm$ $1.70, \mathrm{P}<0.01)$. The prevalence of frailty and prefrailty were significantly different in the constipation group than the nonconstipation group (55\% and $30 \%$ vs. $25 \%$ and $36 \%, \mathrm{P}<0.01$ ).

\section{Comparison of constipation diagnostic criteria items and BSFS between the constipation and non-constipation groups}

Figure 3 compares the constipation diagnostic criteria of the two groups. The frequency of all items was significantly higher

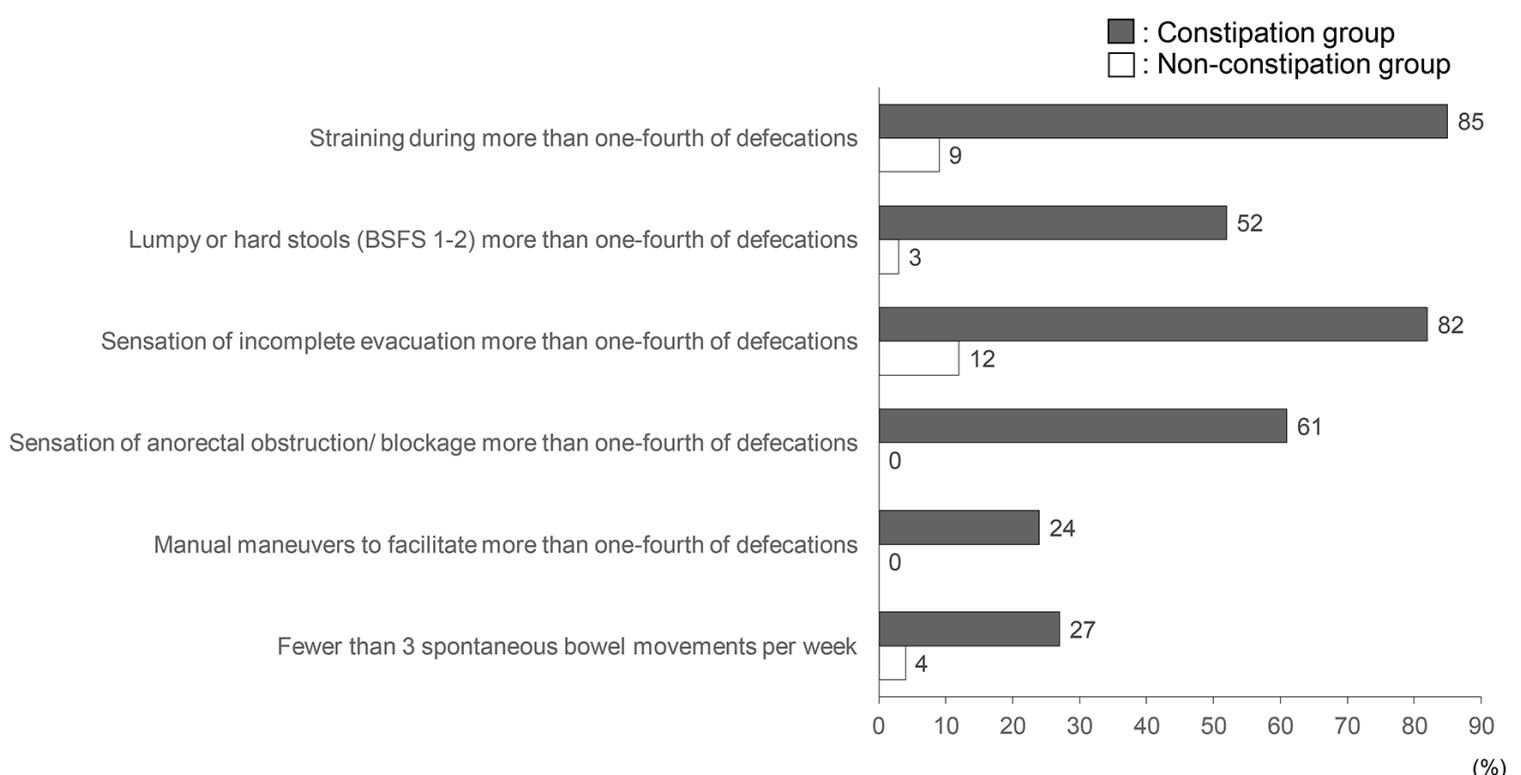

Figure 3. Comparison of diagnostic criteria for functional constipation between the constipation and non-constipation groups. 


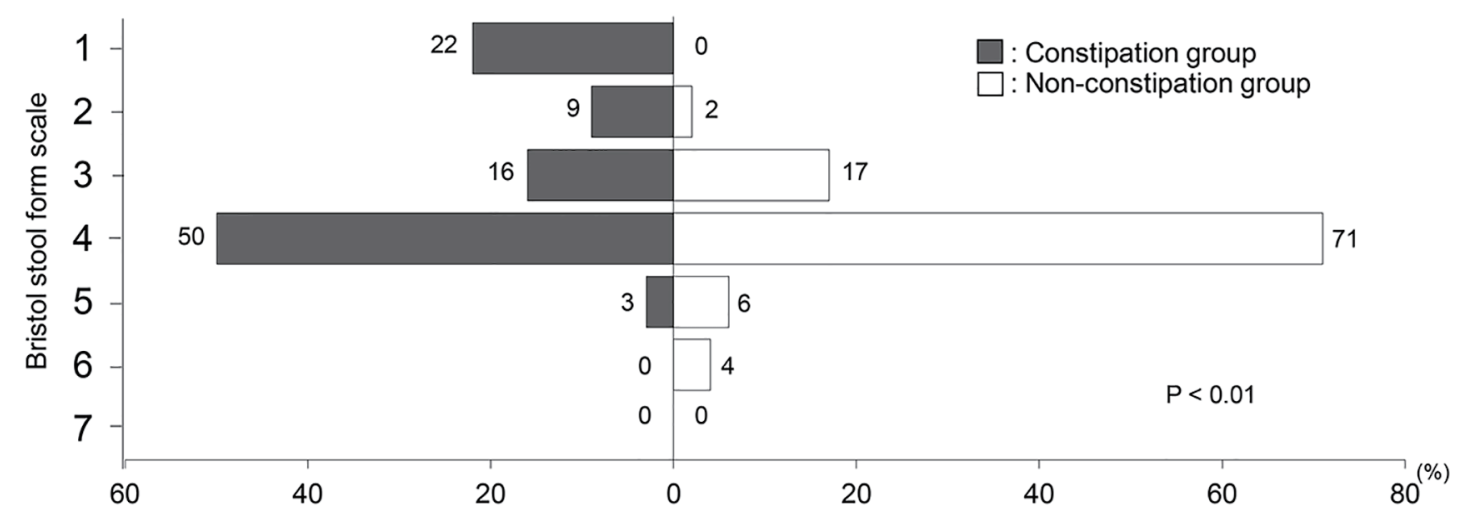

Figure 4. Comparison of Bristol Stool Form Scale between the constipation and non-constipation groups.

in the constipation group than in the non-constipation group (all $\mathrm{P}<0.01)$. In the constipation group, the most common complaints were "straining during over $25 \%$ of defecations" $(85 \%)$ and "sensation of incomplete evacuation during over $25 \%$ of defecations" (82\%). In the non-constipation group, no study subjects responded to "sensation of anorectal obstruction/blockage during more than one-fourth of defecations" or "manual maneuvers to facilitate more than one-fourth of defecations".

The significant differences between the BSFS criteria of the two groups are presented in Figure 4. Types 1 and 2 (hard stools) were observed significantly more often in the constipation group than in the non-constipation group (31\% vs. $2 \%, \mathrm{P}<$ 0.01 ). Types 3 and 4 (ideal stools) were observed significantly more often in the non-constipation group than in the constipation group ( $88 \%$ vs. $66 \%, \mathrm{P}<0.01)$.

\section{Discussion}

This study found that: 1) the prevalence of constipation in patients undergoing CR was relatively higher than the rate reported in Japanese national survey; 2) dissociation was observed between constipation complaint rate and prevalence rate in patients undergoing late phase II CR; and 3) an association was observed between constipation and frailty components. To the best of our knowledge, this is the first report showing the association between constipation and frailty components in patients undergoing late phase II CR.

Constipation is a common digestive disorder associated with lifestyle factors, such as stress and unhealthy diets [13]. The Japan Collaborative Cohort Study demonstrated that constipation could be a marker of exposure to CVD risk factors, which included diabetes, perceived mental stress, depression and sedentary life [13]. The relationship between constipation and cardiovascular events is important, and constipation can be a causative factor for the occurrence of cardiovascular events. In the Japanese population, a lower defecation frequency was associated with a risk of CVD mortality [14]. Subjects with symptoms of chronic constipation and laxative use had a decreased overall survival rate [15]. In addition, excessive straining can cause syncope or cardiac ischemia through cerebral and coronary circulation. Straining causes elevation of blood pressure and can cause cardiovascular events such as congestive heart failure, arrhythmias, acute coronary syndrome and aortic dissection [3].

According to the 2016 comprehensive survey of living conditions conducted by the Japanese Ministry of Health, Labour and Welfare, the prevalence of constipation in Japan is $2-5 \%$; more women are affected than men (4.6\% vs. $2.5 \%$ ) [2]. The prevalence of constipation increases with age [2]; constipation can also be caused by reduced mobility, certain medications, related comorbidities and rectal sensory-motor hypofunction $[16,17]$. Physical inactivity is a major cause of most chronic diseases, including CVD and constipation [17].

In this study, the prevalence of constipation in patients with CVD was significantly higher than the rate found in the Japanese national survey. Various factors, such as diet and physical activity, can contribute to stool hardening and poor bowel movements. Patients with CVD, particularly those with heart failure, are prescribed diuretics and are instructed to limit fluid intake. These can promote constipation.

The Acute Decompensated Heart Failure Syndromes registry, a study of hospitalized heart failure patients in Japan, found that approximately $70 \%$ of patients had fluid retention, and $76 \%$ of patients were administered diuretics in hospital [18]. In patients with heart failure, gastrointestinal disorders were associated with several factors, such as hypoperfusion, abnormality of intestinal permeability and increase of intestinal bacterial colonization [19]. There was no significant difference of types and dosages of diuretics between the constipation and non-constipation groups. However, $66 \%$ of subjects in this study were taking diuretics. The high rate of prescription of diuretics may explain the higher prevalence of constipation in patients undergoing late phase II CR than those in the Japanese national survey. In patients with acute coronary syndrome, hypoactivity of the vagal nerve and increased sympathetic nerve activity are associated with constipation [20]. Acute management of aortic dissection requires bed rest of longer durations than those required in other CVDs. Hence, constipation is more likely to develop in patients with aortic dissection [21]. Elevated blood pressure during defecation can induce cardiac rupture, recurrence of aortic dissection, acute heart failure due to increased cardiac afterload and arrhythmia [3]. A dissociation was observed between the complaint rate 
and the prevalence of constipation in this study. Approximately $30 \%$ of patients reported some constipation symptoms despite being prescribed laxatives. This suggests that our clinical staff needs to use a formal assessment method to investigate the patient's defecation.

The BSFS could be a useful tool for daily practice [22]. It uses a classification of seven stool types, based on texture and morphology, which correlate with gastrointestinal transit times. Types 1 and 2 are representative of constipation and slow intestinal transit, while types 6 and 7 correlated to accelerated transit and diarrhea [22]. Several endocrine and metabolic disorders may be associated with slow-transit constipation and are present without other significant systemic, gastrointestinal, or neurological disorders [23]. The KCL score for physical function, social activities of daily life and depression was significantly higher in the constipation group than in the non-constipation group. Constipation causes depression, anxiety and decreases the quality of life (QOL) [24]. The impacts of constipation on QOL are as important as those of other common chronic conditions [25]. The symptoms of constipation go beyond the frequency of bowel movements to include other unpleasant side effects such as abdominal pain or bloating [4]. Furthermore, physical and mental weakness, which are components of frailty, may be associated with constipation [16]. The Clinical Guidelines for Chronic Constipation is expected to raise awareness and develop new evidence-based treatments according to a unified evaluation [4].

The present study has several limitations. Firstly, this was a single-center observational study with a small sample size; a study with a larger sample size is needed to confirm these findings. Secondly, this study had a cross-sectional design, and we were unable to prove any causal relationships between constipation and frailty components in patients and whether constipation had developed before frailty progression; these findings warrant further studies. Thirdly, we enrolled a narrow range of patients with CVD undergoing late phase II CR: patients with acute myocardial infarction, chronic heart failure and major vessel disease; the results of this study may not be representative of all patients with CVD. Fourthly, the evaluation of constipation was carried out using a questionnaire. It is possible that the diagnosis of constipation is underestimated. However, the questionnaire items were based on the definition of the Clinical Guidelines for Chronic Constipation. Therefore, its questionnaire must be considered to have a clinical significance.

\section{Conclusions}

The prevalence of constipation in patients undergoing CR was relatively higher than that reported in the Japanese national survey. Constipation was associated with physical function and depression score as components of frailty in patients undergoing CR.

\section{Acknowledgments}

These authors thank all study participants and members who carried out data collection in Cardiovascular Rehabilitation and Fitness.

\section{Financial Disclosure}

This work was supported by a MEXT (Ministry of Education, Culture, Sports, Science and Technology)-Supported Program for the Strategic Research Foundation at Private Universities, Japan and a Grant-in-Aid for Scientific Research from the Japan Society for the Promotion of Science, Japan (19K11374, 17K01470).

\section{Conflict of Interest}

The authors do not have any conflict of interest to declare concerning the present article.

\section{Informed Consent}

All subjects provided written informed consent.

\section{Author Contributions}

MN-Y has designed and performed the study. MN-Y, KS and TM have drafted the manuscript and did critical editing. MY, AH, MK, YS, KF, TM, RM, AA, AS, TY, TA, AA, MS, TS, TT and HD have assisted and supported in sample collection. $\mathrm{MN}-\mathrm{Y}$ and KS have carefully supervised this manuscript preparation and writing.

\section{Data Availability}

Any inquiries regarding supporting data availability of this study should be directed to the corresponding author.

\section{References}

1. Shimokawa N, Talley J, O'Keefe EA, Zinsmeister AR, Melton LJ, 3rd. Prevalence of gastrointestinal symptoms in the elderly: a population-based study. Gastroenterology. 1992;102:895-901.

2. The Ministry of Health, Labour and Welfare. Comprehensive survey of living conditions. https://www.mhlw.go.jp/ toukei/saikin/hw/k-tyosa/k-tyosa16/. Accessed on March 8, 2019.

3. Ishiyama Y, Hoshide S, Mizuno H, Kario K. Constipation-induced pressor effects as triggers for cardiovascular events. J Clin Hypertens (Greenwich). 2019;21(3):421425.

4. Japan Society of Gastroneterology-affiliated Study Group, Chronic Constipation Diagnosis and Treatment Study Group, ed. Clinical guidelines for chronic consti- 
pation 2017. Tokyo: Nankodo; 2017 (in Japanese).

5. The Japanese Circulation Society. Guidelines for rehabilitation in patients with cardiovascular disease (JCS 2012). http://www.j-circ.or.jp/guideline/pdf/JCS2012_nohara_ h.pdf. Accessed on May 31, 2018 (in Japanese).

6. Nishitani M, Shimada K, Sunayama S, Masaki Y, Kume A, Fukao K, Sai E, et al. Impact of diabetes on muscle mass, muscle strength, and exercise tolerance in patients after coronary artery bypass grafting. J Cardiol. 2011;58(2):173-180.

7. Fukai K, Sugita A, Tanaka M. A developmental study of the Japanese version of the constipation assessment scale. Kangokenkyu. 1995;28:25-31. (in Japanese) (Abstract in English).

8. Heaton KW, Ghosh S, Braddon FE. How bad are the symptoms and bowel dysfunction of patients with the irritable bowel syndrome? A prospective, controlled study with emphasis on stool form. Gut. 1991;32(1):73-79.

9. Satake S, Senda K, Hong YJ, Miura H, Endo H, Sakurai T, Kondo I, et al. Validity of the Kihon Checklist for assessing frailty status. Geriatr Gerontol Int. 2016;16(6):709715.

10. Kunimoto M, Shimada K, Yokoyama M, Matsubara T, Aikawa T, Ouchi S, Shimizu M, et al. Relationship between the Kihon Checklist and the clinical parameters in patients who participated in cardiac rehabilitation. Geriatr Gerontol Int. 2019;19(4):287-292.

11. Matsuo S, Imai E, Horio M, Yasuda Y, Tomita K, Nitta $\mathrm{K}$, Yamagata $\mathrm{K}$, et al. Revised equations for estimated GFR from serum creatinine in Japan. Am J Kidney Dis. 2009;53(6):982-992.

12. Sugita Y, Miyazaki T, Shimada K, Shimizu M, Kunimoto M, Ouchi S, Aikawa T, et al. Correlation of nutritional indices on admission to the coronary intensive care unit with the development of delirium. Nutrients. 2018;10(11):1712.

13. Kubota Y, Iso H, Tamakoshi A. Bowel movement frequency, laxative use, and mortality from coronary heart disease and stroke among Japanese men and women: the Japan Collaborative Cohort (JACC) study. J Epidemiol. 2016;26(5):242-248.

14. Honkura K, Tomata Y, Sugiyama K, Kaiho Y, Watanabe T, Zhang S, Sugawara Y, et al. Defecation frequency and cardiovascular disease mortality in Japan: The Ohsaki cohort study. Atherosclerosis. 2016;246:251-256.
15. Chang JY, Locke GR, 3rd, McNally MA, Halder SL, Schleck CD, Zinsmeister AR, Talley NJ. Impact of functional gastrointestinal disorders on survival in the community. Am J Gastroenterol. 2010;105(4):822-832.

16. De Giorgio R, Ruggeri E, Stanghellini V, Eusebi LH, Bazzoli F, Chiarioni G. Chronic constipation in the elderly: a primer for the gastroenterologist. BMC Gastroenterol. 2015;15:130.

17. Booth FW, Roberts CK, Laye MJ. Lack of exercise is a major cause of chronic diseases. Compr Physiol. 2012;2(2):1143-1211.

18. Sato N, Kajimoto K, Keida T, Mizuno M, Minami Y, Yumino D, Asai K, et al. Clinical features and outcome in hospitalized heart failure in Japan (from the ATTEND Registry). Circ J. 2013;77(4):944-951.

19. Romeiro FG, Okoshi K, Zornoff LA, Okoshi MP. Gastrointestinal changes associated to heart failure. Arq Bras Cardiol. 2012;98(3):273-277.

20. Schwartz PJ, Vanoli E, Stramba-Badiale M, De Ferrari GM, Billman GE, Foreman RD. Autonomic mechanisms and sudden death. New insights from analysis of baroreceptor reflexes in conscious dogs with and without a myocardial infarction. Circulation. 1988;78(4):969-979.

21. Januzzi JL, Isselbacher EM, Fattori R, Cooper JV, Smith DE, Fang J, Eagle KA, et al. Characterizing the young patient with aortic dissection: results from the International Registry of Aortic Dissection (IRAD). J Am Coll Cardiol. 2004;43(4):665-669.

22. Saad RJ, Rao SS, Koch KL, Kuo B, Parkman HP, McCallum RW, Sitrin MD, et al. Do stool form and frequency correlate with whole-gut and colonic transit? Results from a multicenter study in constipated individuals and healthy controls. Am J Gastroenterol. 2010;105(2):403411.

23. Bharucha AE, Pemberton JH, Locke GR, 3rd. American Gastroenterological Association technical review on constipation. Gastroenterology. 2013;144(1):218-238.

24. Cheng C, Chan AO, Hui WM, Lam SK. Coping strategies, illness perception, anxiety and depression of patients with idiopathic constipation: a population-based study. Aliment Pharmacol Ther. 2003;18(3):319-326.

25. Belsey J, Greenfield S, Candy D, Geraint M. Systematic review: impact of constipation on quality of life in adults and children. Aliment Pharmacol Ther. 2010;31(9):938949. 